\title{
A Two-Stage Multi-objective Programming Model to Improve the Reliability of Solution
}

\author{
Chenxia Jin ${ }^{1,2}$, Fachao $\mathrm{Li}^{2}{ }^{2}$, Kaixin Feng ${ }^{2}$, Yunfeng Guo ${ }^{2}$ \\ ${ }^{1}$ School of Mathematical Sciences, Hebei Normal University, Shijiazhuang 050024, China \\ ${ }^{2}$ School of Economics and Management, Hebei University of Science and Technology, Shijiazhuang 050018, China
}

\author{
ARTICLE INFO \\ Article History \\ Received 15 Oct 2019 \\ Accepted 04 Apr 2020

\section{Keywords} \\ Multi-objective programming \\ Randomness \\ Expectation value \\ Fuzzy events \\ Reliability
}

\begin{abstract}
Randomness is a common uncertainty encountered in practical multi-objectives decision-making. But it is always a challenge for decision-makers to process randomness in multi-objective programming problems. This paper takes the decision-making objectives as fuzzy events and aims to solve numerical multi-objective programming problems under random environment. We first analyze the effects of randomness on multi-objective decision-making results. With the expectation value and the probability of fuzzy events as quantitative index of randomness, we then establish a two-stage random multi-objective programming model based on reliability (i.e., TS-MOPM). Specifically, we give several probability calculation methods of fuzzy events with common distributions, and further present the corresponding calculation procedures for solving TS-MOPM. Finally, a case study is implemented to test the proposed model TS-MOPM. Theoretical analysis and case study indicate that our model has better interpretability and operability. The research results enrich the existing random multi-objective programming methods to some extent.
\end{abstract}

(c) 2020 The Authors. Published by Atlantis Press SARL. This is an open access article distributed under the CC BY-NC 4.0 license (http://creativecommons.org/licenses/by-nc/4.0/).

\section{INTRODUCTION}

In today's real world, complexity has considerably raised for decision-makers because of the increasing uncertainties they are facing. A variety of decision-making problems often involve various objectives to be achieved at the same time. For example, water quality planning and management may refer to factors such as the implementation levels, unit costs, reduction efficiency and total pollution loads [1,2]; emergency relief management considers several different objectives as logistics time, logistics costs, the amount of unsatisfied demand, service delays, the ability of sustainable rescue $[3,4]$; transportation network should balance three indices transportation cost, transportation time and emissions to optimize the system performance [5]; agricultural water and land optimization allocation is a complicated problem involving many objectives [6]; suppliers selection in supply chain management involves some objectives such as cost minimization, the quality of raw materials, on-time delivery and sustainability [7]. In view of the above applications, a multi-objective optimization is the most potential way. Thus, modeling real life problems as formulated multi-objective programming and further giving corresponding solution are two main concerns in management field.

Multi-objective programming is an optimization problem characterized by a set of objective functions and a set of well-defined constraints to be satisfied [8-10]. The goal of multi-objective

*Corresponding author. Email: lifachao@tsinghua.org.cn programming is to identify all the feasible solutions in which it is impossible to optimize the value of one objective function without making the value of at least one other objective worse [11]. Charnes et al. [12] firstly established a multi-objective linear programming model based on bias variables by introducing positive and negative deviation variables and priority factors (including rank and weight). More information on multi-objective programming can be referred to Ehrgott $[9,13]$.

Because many practical decision-making problems can be formulated as a multi-objective programming, the wide application spectrum and ever-increasing strong interests have encouraged the substantial development of reliable models and efficient algorithms during the recent decades [14-17]. Numerous researches have been devoted to multi-objective programming combining with different theories and methods, and many significant theoretical and applied findings have been also achieved. Han et al. [16,17] discussed the solution to multi-objective programming with hierarchical structure (bi/tri-level decision-making). Kao et al. [18] established a multi-objective programming method to solve the network data envelopment analysis model; Dujardin et al. [19] established a multi-objective interactive system for adaptive traffic control; Zhou et al. [20] proposed a selection hyper-heuristic based algorithm for solving multi-objective optimization problems; for the solving algorithm for Multi-objective binary programs, Boland et al. [11] discussed the theory foundation of preprocessing and cut generation techniques guaranteeing the existence of a feasible solution. Pal et al. [21] presented a heuristic based on feasibility pump for 
approximately solving multi-objective mixed integer linear programs. Lokman [22] developed an interactive algorithm for multiobjective integer programs under the requirement of a desired level of accuracy.

Given the interest in the refereed models and algorithms for multiobjective programming, it is surprising that little has considered the randomness in decision-making. Furthermore, randomness is inevitable in the actual decision-making environment. Therefore, many strategies have been proposed to deal with the uncertainties. For example, Liu et al. [23] established an expectation model of stochastic programming with expectation value; Charnes et al. [24] established a chance-constrained model with reliability as the key point of stochastic decision; referring the multiple tasks in the complex system as events, Liu [25] established a dependent-chance programming model through optimizing the probability of events in random environments (i.e., the chance function). The above three models are common in dealing with randomness. Thereafter, a large number of models were proposed by extending these work. Abdelaziz et al. [26] assumed that the parameters related to the target obeyed the normal distribution and proposed a stochastic multi-objective programming portfolio model, then transformed it into an chance-constrained compromise programming model for solution; Li et al. [27] used expectation value and variance as the composite quantitative indicators of random variables, and established the generalized expectation model of stochastic programming; for the uncertainties existed in inventory, transportation cost and demand in the recycling logistics network, Sazvar et al. [28] proposed a stochastic programming model based on expected value; Masri et al. [29] established a multi-objective stochastic programming model based on the retailer's optimal working capital level, and proposed multiple solution strategies based on chance constrained approach, a recourse approach and stochastic goal programming approach; Tong et al. [30] used random variables to describe the target under uncertain demand, established a bi-level programming model of stochastic multi-objective logistics network design and further designed genetic algorithm (GA) based on stochastic simulation for solution; Fard et al. [31] established a twostage stochastic multi-objective programming model for closedloop supply chain (CLSC) considering environmental factors and downside risk. Moreover, a number of memetic metaheuristics had been considered. Rong et al. [1] integrated the global nutrient export from watersheds model, interval parameter programming and stochastic chance-constrained programming into a general simulation-based interval stochastic bi-level multi-objective programming framework. Zhang et al. [4] proposed a multi-objective three-stage stochastic programming model to minimize transportation time, transportation cost and unsatisfied demand in emergency logistics. For the multiple uncertain factors in water resources optimization, Ren et al. [6] developed an improved multi-objective stochastic fuzzy programming method. For the multiple uncertainties in water resources management, Nematian et al. [32] used probability theory to describe fuzzy random variables and then develop an extended multi-objective mixed integer programming approach.

Previous studies on stochastic programming methods only focus on one facet: the existence of decision-making results or the reliability of decision-making results, but did not consider both systematically. Actually, both of them need to be considered in the decision-making process. In this paper, a general multi-objective programming model is proposed to solve the two problems.
This motives us to establish a two-stage multi-objective programming model (TS-MOPM). In the first stage, we aim to minimize the difference between objective function and the target value by introducing the deviation variables and weights based on priority factor. In the second stage, we propose a strategy of maximizing the synthetic realization probability of decision-making objectives to improve the reliability of the results obtained in the first stage. The resulting two-stage model is difficult to solve because of multiple uncertainties (fuzziness and randomness). For this purpose, expectation value and the probability of fuzzy events are separately used to transfer the model to be deterministic. Numerical experiments based on a pharmaceutical factory indicate the effectiveness and advantages of our multi-stage model. Compared with the previous studies, the main contributions of this paper are summarized as follows: 1) TS-MOPM guarantees some feasible solutions by using deviation variables; 2) TS-MOPM deals with multiple uncertainties by expectation value and the probability of fuzzy events; 3) TS-MOPM optimizes the synthetic realization probability of decision-making objectives. In general, a desired high satisfaction level decision-making scheme could be obtained by TS-MOPM.

The remainder of the paper is organized as follows: Section 2 reviews three common multi-objective programming models. In Section 3, a two-stage multi-objective programming model (TSMOPM) is proposed. In Section 4, the solution strategy for TS-MOPM is given. Numerical experiments are performed in Section 5. Conclusions are drawn in Section 6.

\section{OVERVIEW OF MULTI-OBJECTIVE PROGRAMMING MODELS}

A general multi-objective programming model with $s$ objectives can be formulated as follows:

$$
\left\{\begin{array}{l}
\min \left[f_{1}(x), f_{2}(x), \cdots, f_{s}(x)\right] \\
\text { s.t. } g_{i}(x) \geq 0, i=1,2, \cdots, m
\end{array}\right.
$$

In model (1), $x=\left(x_{1}, x_{2}, \cdots, x_{n}\right)$ represents the decision variable; $g_{i}(x) \geq 0, i=1,2, \cdots, m$ represents all the constraints; $f_{1}(x), f_{2}(x), \cdots, f_{s}(x)$ represent $s$ objectives. $f_{k}(x)$ is the $k$ th decision-making objective which can be approximately specified as the combination of an objective function $h_{k}(x)$ and a target value $V_{k}$, i.e., $f_{k}(x)=\left\{h_{k}(x), V_{k}\right\}$. Here, $V_{k}$ can be a certain numerical value, a set or a random variable obeying special probability distribution. Particularly, model (1) degrades to a numerical multi-objective programming when $f_{1}(x), f_{2}(x), \cdots, f_{s}(x)$ and $g_{i}(x), i=1,2, \cdots, m$ are real value functions; model (1) is an uncertain multi-objective programming when $f_{1}(x), f_{2}(x), \cdots, f_{s}(x)$ or $g_{i}(x), i=1,2, \cdots, m$ have some uncertainties.

In some real situations, the decision-making objectives are often conflicting with constraints to a certain degree, which leads to the inexistence of decision-making schemes absolutely making all the objectives optimal. In most cases, there does not exist direct solving methods suitable for model (1). One of the usually adopted methods is to convert model (1) into a single objective programming by some strategies. The common used methods are summarized as follows: 
Method 1: Multi-objective programming based on priority factors [12]. This method was proposed by Charnes and Cooper in 1961. The basic idea is to minimize the deviation between each objective function and each target value. By introducing priority (including priority level and priority weight), model (1) can be transformed into the following programming model:

$$
\begin{aligned}
& \min \sum_{l=1}^{L} q_{l} \sum_{k=1}^{s}\left(w_{l k}^{-} d_{k}^{-}+w_{l k}^{+} d_{k}^{+}\right) \\
& \text {s.t. }\left\{\begin{array}{l}
h_{k}(x)+d_{k}^{-}-d_{k}^{+}=V_{k}, k=1,2, \cdots, s \\
g_{i}(x) \leq b_{i}, i=1,2, \cdots, m \\
d_{k}^{-}, d_{k}^{+} \geq 0, k=1,2, \cdots, s
\end{array}\right.
\end{aligned}
$$

In model (2), $V_{k}$ denotes the target value of the $k$ th decision-making objective; $d_{k}^{+}, d_{k}^{-}$, named the positive and negative deviation variables, denote the positive and negative difference between objective function and target value $V_{k}$, respectively. Correspondingly, $w_{l k}^{-}, w_{l k}^{+}, k=1,2, \cdots, s$ denote the weights of $d_{k}^{+}, d_{k}^{-}$at the lth level; $q_{l}$ denotes the priority level of $k$ th decision-making objective, with the value $\left\{q_{1}, q_{2}, \cdots, q_{L}\right\}$ satisfying $q_{1} \gg q_{2} \gg \cdots \gg q_{L}$. Here, the symbol $a \gg b$ means that $a$ is far greater than $b$.

Model (2) fits for the decision-making problem with numerical target value. When $g_{i}(x), i=1,2, \cdots, m$ and $h_{k}(x), k=1,2, \cdots, s$ are linear functions, model (2) is a linear programming model which can be solved by the simplex method; when any of $g_{i}(x), i=$ $1,2, \cdots, m$ or $h_{k}(x), k=1,2, \cdots, s$ has nonlinear characteristics, model (2) is a nonlinear programming model which can be solved by lingo software. It shows that the model has good solvability. Model (2) allows some deviations in the target value to guarantee the existence of decision-making results. Although model (2) has been successfully applied in many decision-making problems, it still lacks commonality demonstrated as follows: 1) model (2) cannot solve the uncertain programming problems which are the most popular under the practical decision-making environment; 2) The real decision-making problems don't necessarily consider the absolute difference on the objective level. However, the decisionmakers may pay different concern on each objective. Besides, dimensional difference of each objective will directly lead to many difficulties in explaining the implications of the decision-making results.

The real decision-making problems often have certain randomness. Following will review the two common methods for dealing with random decision-making problems.

Method 2: Expectation value model [23]. The basic idea is to use expectation value to represent random objective and constraints approximately. Based on the above idea, the multi-objective expectation value model can be established as follows:

$$
\left\{\begin{array}{l}
\max \left[E\left[f_{1}(x, \xi)\right], E\left[f_{2}(x, \xi)\right], \cdots, E\left[f_{s}(x, \xi)\right]\right] \\
\text { s.t. } E\left[g_{i}(x, \xi)\right] \leq 0, i=1,2, \cdots, m
\end{array}\right.
$$

In model (3), $E(\xi)$ denotes the expectation value of random variable $\xi ; E\left[f_{k}(x, \xi)\right]$ denotes the numerical decision-making objective, some combinations of objective function $E\left[h_{k}(x, \xi)\right]$ and satisfaction value $V_{k}$, i.e., $E\left[f_{k}(x, \xi)\right]=\left\{E\left[h_{k}(x, \xi)\right], V_{k}\right\}, k=$ $1,2, \cdots, s$.
Through transforming random programming problem into deterministic one using expectation value, model (3) makes up for the deficiencies of model (2) which cannot deal with uncertainties. Because the values of the variables have randomness, the expectation value cannot effectively describe the value characteristics of the random variables when the variance is greater, and the reliability of decision results is difficult to guarantee. For example, $E(\xi)=3.5$ when $\xi$ obeys a uniform distribution on $[2,5]$. At this time, the difference between each value of random variable $\xi$ and its expectation value $E(\xi)$ is small, so it is more appropriate to describe the random variable using the expectation value. However, $E(\xi)=51$ when $\xi$ obeys the uniform distribution on $[2,100]$. In random experiments, $\xi$ will take any real number on $[2,100]$ with equal probability. The difference between the values $\xi=100(\xi=2)$ and $E(\xi)=51$ is great. Therefore, it is not appropriate to use expectation value to represent random variables approximately for this case.

Method 3: Chance-constrained model [24]. It was proposed by Charnes and Cooper in 1959. The basic idea is to quantify random objectives and constraints by some reliability thresholds. Its basic form is shown as follows:

$$
\begin{aligned}
& \min \left[\bar{f}_{1}(x), \bar{f}_{2}(x), \cdots, \bar{f}_{s}(x)\right] \\
& \text { s.t. }\left\{\begin{array}{l}
P\left(h_{k}(x, \xi) \leq \bar{h}_{k}(x, \xi)\right) \geq \beta_{k}, k=1,2, \cdots, s \\
P\left(g_{i}(x, \xi) \leq 0\right) \geq \alpha_{i}, i=1,2, \cdots, m
\end{array}\right.
\end{aligned}
$$

In model (4), $P(A)$ denotes the probability of $A, \beta_{k}$ denotes the satisfaction threshold of the $k$ th decision objective, $\alpha_{i}$ denotes the satisfaction threshold of constrains $g_{i}(x) \leq 0, \bar{f}_{k}(x)$, specified as a combination of objective function $\bar{h}_{k}(x, \xi)$ and satisfaction value $V_{k}$, denotes the numerical objective, which can represented as a form of $\bar{f}_{k}(x)=\left\{\bar{h}_{k}(x), V_{k}\right\}, k=1,2, \cdots, s$.

When the random variables have great randomness, model (4) can control the decision-making quality to further guarantee the reliability through presetting the threshold. Therefore, model (4) makes up for the deficiencies existed in model (3) to a certain extent. However, the computational complexity of model (4) is large if too many objectives are taken into consideration or the decisionmaking environment is much complicated. Besides, there does not exist an easy solution method. Specifically, the decision-making satisfying all the constraints " $P\left(g_{i}(x) \leq 0\right) \geq \alpha_{i}, i=1,2, \cdots, m$ " simultaneously may not exist.

Combined with the above discussions, many scholars have established various multi-objective programming models based on fuzzy set theories, stochastic theories and effect theories, and also achieved better application results. However, most of these models focus on either the existence of solutions or the reliability of decision-making results, and do not systematically fusion the two aspects. The existence of decision-making results decides whether a plan can be implemented, and the reliability can reflect the decision-making quality, both of which should be considered in the decision-making process. Therefore, establishing a multi-objective programming method considering the existence and the reliability of the results comprehensively is of vital value and practical significance. 


\section{TS-MOPM BASED ON RELIABILITY}

In this section, we will establish a two-stage multi-objective random programming model that can measure the reliability of decisionmaking results, its general framework consists of two modules as shown in Figure 1.

The first stage model: Considering that the practical decisionmaking allows the target value to deviate within the limits. Our model will introduce the deviation variables to describe the difference between the objective function and the target value. Additionally, decision-makers may give different attentions (preferences) on the objectives, but these attentions still cannot reach obvious level difference. Therefore, our model will give different weights to different objectives. For the randomness in modeling, expectation value can be used to process random variables. Based on the above discussions the idea of model (3), the first stage model (expectation value multi-objective programming model) can be formulated as follows:

$$
\begin{aligned}
& \min z=\sum_{k=1}^{s}\left(w_{k}^{-} d_{k}^{-}+w_{k}^{+} d_{k}^{+}\right) \\
& \text {s.t. }\left\{\begin{array}{l}
E\left(h_{k}(x, \xi)\right)+d_{k}^{-}-d_{k}^{+}=V_{k}, k=1,2, \cdots, s \\
E\left(g_{i}(x, \xi)\right) \leq b_{i}, i=1,2, \cdots, p \\
g_{j}(x) \leq b_{j}, j=1,2, \cdots, q \\
d_{k}^{-}, d_{k}^{+} \geq 0, k=1,2, \cdots, s
\end{array}\right.
\end{aligned}
$$

Stage 1: Expectation value multi-objective programming model

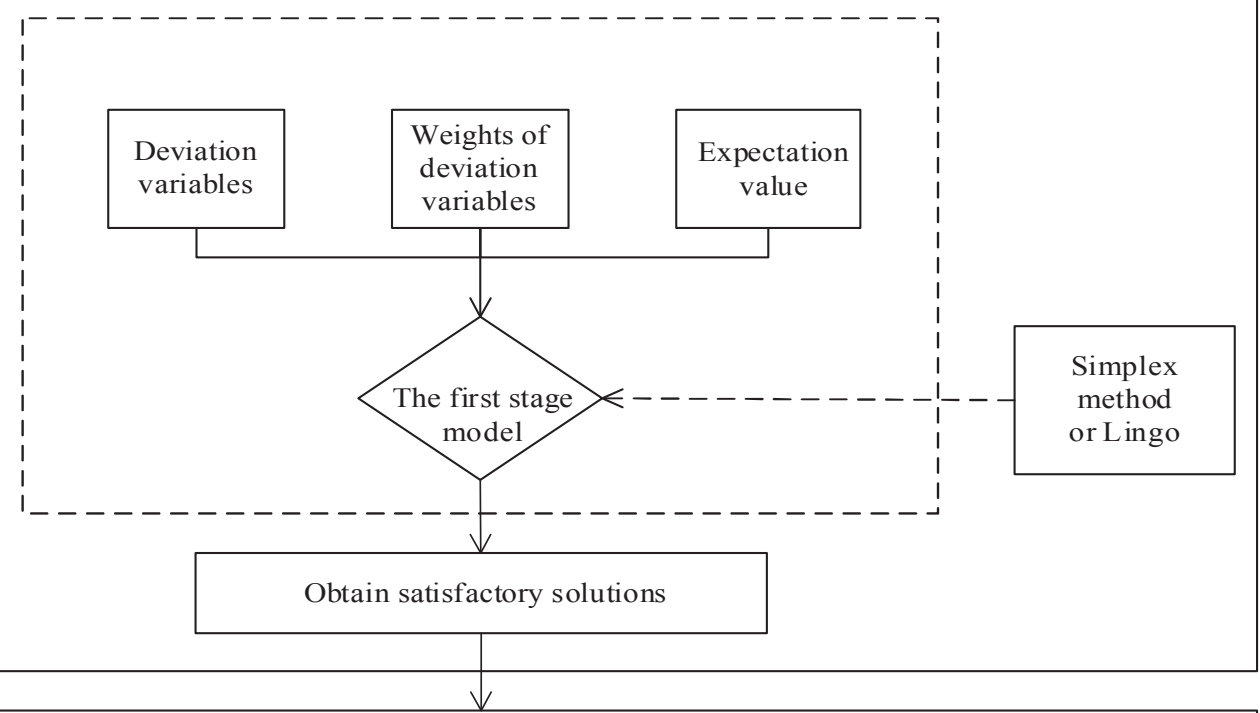

Stage2: Multi-objective programming model based on probability

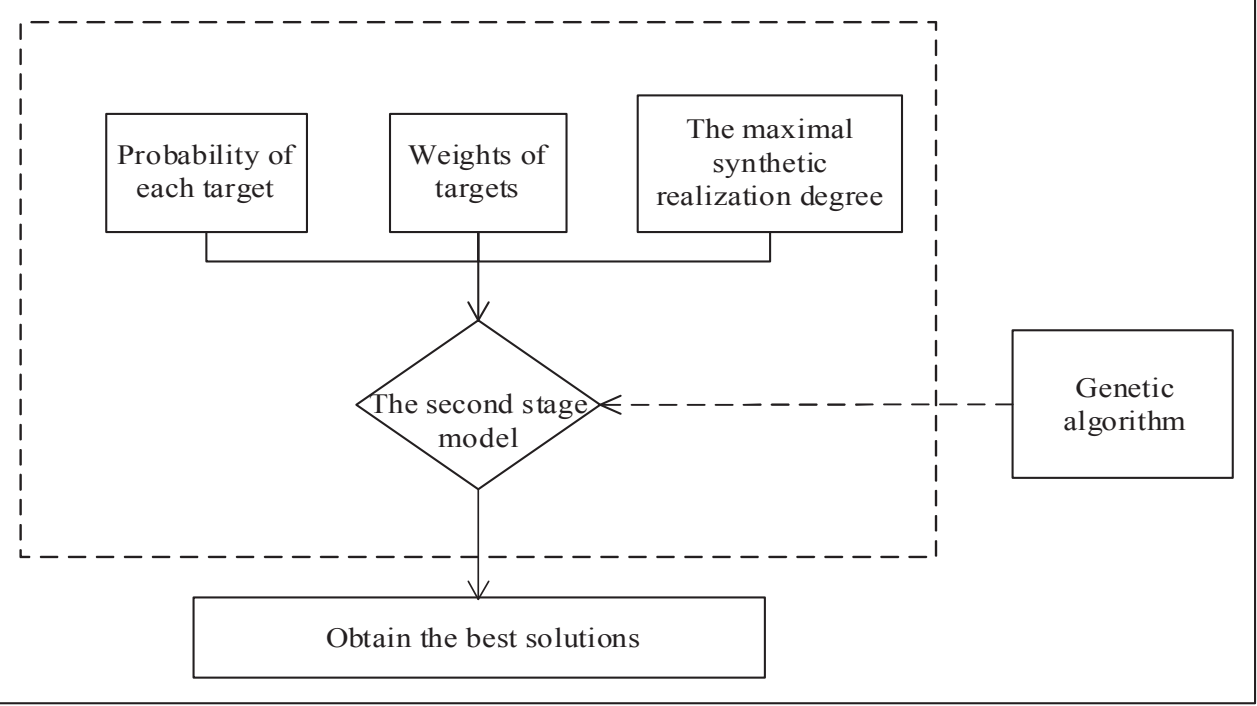

Figure 1 An overall process of two-stage random multi-objective programming model based on reliability (TS-MOPM). 
In model (5), $E\left(h_{k}(x, \xi)\right)$ denotes the expectation value of the $k$ th objective function including random variables, $g_{j}(x) \leq$ $b_{j}, j=1,2, \cdots, q$ denotes numerical constraints, $E\left(g_{i}(x, \xi)\right) \leq$ $b_{i}, i=1,2, \cdots, p$ denotes the constraints including random variables.

Model (5), a combination of model (2) and (3), has the following features: (1) It can deal with uncertain programming problems by transformation; (2) It can be solved directly by simplex method or lingo and has better solvability; (3) The existence of solution can be guaranteed. The fact that the target value allows some deviations demonstrates that a satisfactory solution can be obtained. However, model (5) still has some shortcomings: (1) When the decisionmaking objectives have different dimensions, it is very difficult to give the reasonable explanation for the decision-making results; (2) Model (5) only considers the expectation value of random variables, but missing the influence of variance. When the variables present great randomness, model (5) cannot guarantee the reliability of the results.

The second stage model: Under the framework of model (5), these expressions on real decision-making objectives, such as "Profits should not be less than $a$ million yuan; Equipment occupy time should be $b$ hours as much as possible; The raw material consumption should not be more than $c$ kilogram as possible," are the most common. Obviously, they have fuzziness and can be described as fuzzy events. For this case, it is necessary to deal with randomness and fuzziness simultaneously for solution. Probability theories are often used to deal with randomness. For instance, some scholars discussed the probability calculation method of fuzzy events with the aids of fuzzy set theories [33]. Zadeh [34] firstly proposed a probability calculation model of numerical fuzzy events of probability space with membership degree as the action coefficients. This method has good interpretability, but it cannot realize the probability calculation of algebraic operations of fuzzy events. Under the background of cognition inconsistency, Yager [35,36] proposed a fuzzy probability description strategy of fuzzy events, and gave two kinds computation methods based on cut sets. Based on the decomposition theorem of fuzzy sets and the probability of every cut set, Chen et al. [37] gave a probability calculation method of fuzzy events by using integral as a synthesis operator. Considering that decision-makers would show fuzzy preferences and each membership state would have nonlinear effect on the decision process, Li et al. [38] introduced the level effect function and further established an effect probability calculation method of fuzzy events.

For the fuzzy target value $V_{k}$ under random environment, we introduce the probability of fuzzy events to describe the realization degree of objectives, then the objective function can be expressed as $P\left(h_{k}(x, \xi) \in V_{k}\right) \triangleq p_{k}(x)$, where $p_{k}(x)$ represents the decisionmaking objective with objective function $h_{k}(x, \xi)$ and satisfaction value 1 . In the first stage model, some satisfactory solutions are sure to obtain. However, the reliability of solutions cannot be guaranteed. In order to judge whether there exist some solutions with even higher satisfaction degree, we take the synthetic satisfaction degree of the solutions obtained in the first stage as a constraint. Further, by maximizing the synthetic realization probability of decisionmaking objectives, the second stage model (multi-objective programming model based on probability) can be established as follows:

$$
\begin{aligned}
& \max p(x)=\sum_{k=1}^{s} w_{k} p_{k}(x) \\
& \text { s.t. }\left\{\begin{array}{l}
p_{k}(x)=P\left(h_{k}(x, \xi) \in V_{k}\right), k=1,2, \cdots, s \\
E\left(g_{i}(x, \xi)\right) \leq b_{i}, i=1,2, \cdots, p \\
g_{j}(x) \leq b_{j}, j=1,2, \cdots, q \\
\sum_{k=1}^{s} w_{k} p_{k}(x) \geq \beta
\end{array}\right.
\end{aligned}
$$

In model (6), the importance weights of the targets value satisfy $\sum_{k=1}^{s} w_{k}=1$; when the satisfactory solution $x_{0}$ obtained in the first stage is unique, $\beta$ indicates the synthetic realization degree of each decision-making objective based on $x_{0}$, and $\beta=\sum_{k=1}^{s} w_{k} p_{k}\left(x_{0}\right)$; when the satisfactory solution $x_{0}$ obtained in the first stage is not unique, $\beta$ is the maximal synthetic realization degree and $\beta=\max \sum_{k=1}^{s} w_{k} p_{k}\left(x_{0}\right)$. Obviously, the greater (smaller) the synthetic realization probability $\sum_{k=1}^{s} w_{k} p_{k}(x)$ is, the greater (smaller) the degree of the objective function reaching the target value is, the higher (lower) the reliability of the decision-making results is, the greater (smaller) the recognition degree of the decision-making results is.

Obviously, model (6) can make up for the deficiencies of model (5) to a certain extent, which can be stated as follows: (1) Model (6) is a dimensionless model. It can better respond the uninterpretability of decision-making results caused by different target dimensions. (2) Model (6) has good structural characteristics. Probability, a descriptive form of decision-making objectives, can directly reflect the realization degree of the target. Additionally, taking the synthetic realization degree of the first stage objective as a constraint, model (6) can not only reduce the range of solution, but also deduce whether there exists a solution with even higher reliability. (3) Model (6) has good interpretability. It can help decision-makers get the synthetic realization degree of the results under different conditions and the realization degree of each objective, which is very useful in explaining the implications of decision-making results.

Remark 1. Model (6) aims at maximizing the synthetic realization degree of all the targets, without considering the realization degree of each single target. In particular, the constraints $P\left(h_{k}(x, \xi) \in V_{k}\right) \geq \alpha_{k}, k=1,2, \cdots, s$ can be applied to model (6) for special requirements for the realization degree of some targets. Where, $\alpha_{k}$ is the lowest realization degree of the $k$ th objective (i.e., the satisfaction threshold).

TS-MOPM based on reliability improves expectation value model (3) and chance-constrained model (4) to a certain extent. It guarantees the existence of decision-making results, also considers the reliability at the same time. Therefore, TS-MOPM has better theoretical and instructive significance for decision-making problems under random environments.

\section{SOLUTION STRATEGY FOR TS-MOPM}

This section will discuss the probability calculation method of fuzzy event for several common distributions, and give the specific solution procedures of TS-MOPM. 
In the following, we assume that

1. Let $(\Omega, \mathcal{B}, P)$ be a probability space. Where, $\mathcal{B}$ is a $\sigma$-algebra constituted by some subsets of $\Omega ; P: \mathcal{B} \rightarrow[0,1]$ satisfying: (1) $P(\phi)=0, P(\Omega)=1$; (2) $P\left(\cup_{n=1}^{\infty} A_{i}\right)=\sum_{n=1}^{\infty} P\left(A_{i}\right)$ for $\left\{A_{n}\right\}_{n=1}^{\infty} \subset \mathcal{B}$ and any two of them are incompatible.

2. Let $\mathcal{F}(\Omega)$ be a family of fuzzy sets on $\Omega$, i.e., a mapping from $\Omega$ to $[0,1]$.

3. For $A \in \mathcal{F}(\Omega)$, let $A(x)$ be the membership function of $A$, and $A_{\lambda}=\{x \mid A(x) \geq \lambda\}$ be the $\lambda$-cut set.

4. If $A$ satisfies: (1) $A_{1} \neq \varnothing$; (2) $A_{\lambda}$ is bounded closed interval for any $\lambda \in[0,1]$; (3) $\operatorname{supp} A=\{x \mid A(x)>0\}$ is bounded, then $A$ is called a fuzzy number. Especially, if there exist real numbers $a, b, c$ satisfying: (1) $A(x)=0$ for any $x<a$ or $x>c$; (2) $A(x)=(x-a) /(b-a)$ for any $a \leq x \leq b$; (3) $A(x)=(c-x) /(c-b)$ for any $b<x \leq c$, then $A$ is called a triangular fuzzy number, and denoted as $A=(a, b, c)$ for short.

\subsection{Probability Calculation of Fuzzy Events}

This paper will use probability calculation model of [37], which is a special case of [38] for level effect function $L(\lambda)=1$.

Definition 1. [37] Let $(\Omega, \mathcal{B}, P)$ be a probability space and $A$ be a fuzzy event on $A=(a, b, c)$, then

$$
P(A)=\int_{0}^{1} P\left(A_{\lambda}\right) d \lambda
$$

is called the probability of $A$. Here, $P\left(A_{\lambda}\right)$ can be understood as "the probability of an event whose degree of satisfying $A$ is more than or equal to $\lambda$."

If we take the decision-making objective as fuzzy events, then the fuzziness of the target value can be described as fuzzy numbers, the most used fuzzy information description tool is triangular fuzzy number. Combining triangular fuzzy number, Eq. (7) with common distributions, for any continuous random variable $X$ on probability space $(\Omega, \mathcal{B}, P)$, we have,

$$
P(X \in A)=\int_{0}^{1} \int_{\underline{a}(\lambda)}^{\bar{a}(\lambda)} \varphi(x) d x d \lambda
$$

In Eq. (8), $\varphi(x)$ is the probability density function, $A_{\lambda}=$ $[\underline{a}(\lambda), \bar{a}(\lambda)]$. If $\varphi(x)$ and $A$ are given, Eq. (8) is an ordinary double integral with fixed form. Therefore, Eq. (8) is a general calculation model. We will discuss the properties of Eq. (8) for triangular fuzzy number $A=(a, b, c)$.

Theorem 1. Let $X$ be continuous random variables on $(\Omega, \mathcal{B}, P), A=$ $(a, b, c)$. Then

$$
P(X \in A)=P(X \in(a, b, b))+P(X \in(b, b, c)) .
$$

Proof: According to Eq. (8) and $A_{\lambda}=[a+(b-a) \lambda, c-(c-b) \lambda]$, we know

$$
\begin{aligned}
P(X \in A) & =\int_{0}^{1} \int_{a+(b-a) \lambda}^{c-(c-b) \lambda} \varphi(x) d x d \lambda \\
& =\int_{a}^{b} \int_{0}^{\frac{x-a}{b-a}} \varphi(x) d \lambda d x+\int_{b}^{c} \int_{0}^{\frac{c-x}{c-b}} \varphi(x) d \lambda d x \\
& =P(X \in(a, b, b))+P(X \in(b, b, c)) .
\end{aligned}
$$

Combined with several common distributions, we have the following calculation formula based on Eq. (8) and Theorem 1.

Theorem 2. Let $X$ be continuous random variables on $(\Omega, \mathcal{B}, P), A=$ $(a, b, c)$. Then we have,

1. If $X$ obeys uniform distribution $U\left[\theta_{1}, \theta_{2}\right], \theta_{1} \leq a \leq$ $c \leq \theta_{2}$, and the probability density function is $\varphi(x)=$ $\left\{\begin{array}{ll}\left(\theta_{2}-\theta_{1}\right)^{-1}, & \theta_{1}<x<\theta_{2} \\ 0, & x \leq \theta_{1} \text { or } x \geq \theta_{2}\end{array}\right.$, then $P(X \in A)=\frac{c-a}{2\left(\theta_{2}-\theta_{1}\right)}$.

2. If $X$ obeys exponential distribution $\operatorname{Exp}(\theta)$ with parameter $\theta, a \geq$ 0 , and the probability density function is $\varphi(x)=\left\{\begin{array}{ll}\theta e^{-\theta x}, & x \geq 0 \\ 0, & x<0\end{array}\right.$, then $P(X \in A)=\frac{e^{-\theta a}-e^{-\theta b}}{\theta(b-a)}+\frac{e^{-\theta c}-e^{-\theta b}}{\theta(c-b)}$.

3. If $X$ obeys standard normal distribution $N(0,1)$, and the probability density function is $\varphi(x)=\frac{1}{\sqrt{2 \pi}} e^{-\frac{x^{2}}{2}}$, then $P(X \in A)=$ $G(a, b)+G(-c,-b)$. Here, $G(\alpha, \beta)=\frac{1}{\beta-\alpha} \int_{\alpha}^{\beta}(x-\alpha)$. $\frac{1}{\sqrt{2 \pi}} e^{-\frac{x^{2}}{2}} d x$

4. If $X$ obeys normal distribution $N\left(\mu, \sigma^{2}\right)$, and the probability density function is $\varphi(x)=\frac{1}{\sqrt{2 \pi} \sigma} e^{-\frac{(x-\mu)^{2}}{2 \sigma^{2}}}$, then $P(X \in(a, b, c))=$ $G\left(\frac{a-\mu}{\sigma}, \frac{b-\mu}{\sigma}\right)+G\left(\frac{\mu-c}{\sigma}, \frac{\mu-b}{\sigma}\right)$. Here, $G(\alpha, \beta)=\frac{1}{\beta-\alpha} \int_{\alpha}^{\beta}(x-\alpha)$. $\frac{1}{\sqrt{2 \pi}} e^{-\frac{x^{2}}{2}} d x$.

Remark 2. In practical decision-making, the results changing within a certain range of the target value are acceptable. However, the satisfaction degree will decrease with the deviation from the target value increasing. Therefore, this paper introduces the deviation parameter $\delta_{i}, i=1,2,3$ (the maximum value allowed to deviate from the target value). In the following, we will give three common decision-making objectives under fuzzy environment with the membership function,

1. $X_{1}$ is not more than $V_{1}$ as possible, then $A\left(X_{1}\right)=$ $\begin{cases}1, & X_{1} \leq V \\ \left(V_{1}+\delta_{1}-X_{1}\right) / \delta_{1}, & V_{1}<X_{1}<V_{1}+\delta ; \\ 0, & X_{1} \geq V_{1}+\delta_{1}\end{cases}$ 
2. $X_{2}$ is not less than $V_{2}$ as possible, then $A\left(X_{2}\right)=$

$$
\begin{cases}0, & X_{2} \leq V_{2}-\delta_{2} \\ \left(X_{2}-V_{2}+\delta_{2}\right) / \delta_{2}, & V_{2}-\delta_{2}<X_{2}<V \\ 1, & X_{2} \geq V_{2}\end{cases}
$$

3. $X_{3}$ is equal to $V_{3}$ as possible, then $A\left(X_{3}\right)=$

$$
\left\{\begin{array}{ll}
\left(X_{3}-V_{3}+\delta_{3}\right) / \delta_{3}, & V_{3}-\delta_{3}<X_{3}<V_{3} \\
\left(V_{3}+\delta_{3}-X_{3}\right) / \delta_{3}, & V_{3} \leq X_{3}<V_{3}+\delta_{3} \\
0, & X_{3} \leq V_{3}-\delta_{3} \text { or } X_{3} \geq V_{3}+\delta_{3}
\end{array} .\right.
$$

Here, $\delta_{i}, i=1,2,3$ reflects the global maximum deviation degree from the target value. Too greater $\delta_{i}$ will lead to decision-making failure. Therefore, the value of $\delta_{i}, i=1,2,3$ can be set according to the absolute deviation of the objectives in the first stage and the acceptance of the decision-makers.

Remark 3. If we set $X$ denote the random variable, $B$ denotes fuzzy events that is not less (more) than $b$ as possible, then the probability of $B$ can be shown as follows, $\delta$ is the maximal allowable limit characterizing the fact "less (more) than $b$."

$$
\begin{aligned}
P(X \in B) & =P(X \in(b-\delta, b, b))+P(X \geq b) \\
& =\int_{0}^{1} \int_{b-(1-\lambda) \delta}^{+\infty} \varphi(x) d x d \lambda \\
& =\int_{b-\delta}^{b} \int_{0}^{\frac{x-(b-\delta)}{\delta}} \varphi(x) d \lambda d x+\int_{b}^{+\infty} \varphi(x) d x
\end{aligned}
$$

$$
\begin{aligned}
P(X \in B) & =P(X \leq b)+P(X \in(b, b, b+\delta)) \\
& =\int_{0}^{1} \int_{-\infty}^{b+(1-\lambda) \delta} \varphi(x) d x d \lambda \\
& =\int_{-\infty}^{b} \varphi(x) d x+\int_{b}^{b+\delta} \int_{0}^{\frac{b+\delta-x}{\delta}} \varphi(x) d \lambda d x
\end{aligned}
$$

The above discussions can provide effective probability calculation of fuzzy events to a certain extent.

\subsection{Solution Procedures of Solving TS-MOPM}

Based on the probability calculation of fuzzy events proposed above, we will provide the complete solution procedures for solving TSMOPM as follows:

[Begin]

Step 1 Obtain the satisfactory solutions of the first stage model (5)

Step 1.1 Set appropriate positive and negative deviation variables $d_{k}^{+}, d_{k}^{-}$and corresponding weights $w_{k}^{+}, w_{k}^{-}$;

Step 1.2 Initialize the expectation value $E\left(h_{k}(x, \xi)\right)$, $E\left(g_{i}(x, \xi)\right) \leq b_{i}, i=1,2, \cdots, p$ according to the distribution of random variables.
Step 1.3 Adopt simplex method or lingo software to obtain the satisfactory solutions $x_{0}$ to model (5). Then go to step 2 .

Step 2 Compute the probability of each target of model (5)

Step 2.1 Set deviation degree $\delta_{k}$ for each target $V_{k}, k=$ $1,2, \cdots, s$, and the triangular fuzzy number representation of each decision-making objective $h_{k}(x, \xi)$;

Step 2.2 Compute the probability $p_{k}\left(x_{0}\right)$ of each target according to Theorem 2 . Then go to step 3 .

Step 3 Set the weights $w_{k}, \sum_{k=1}^{s} w_{k}=1$, compute $\beta=$ $\max \sum_{k=1}^{s} w_{k} p_{k}\left(x_{0}\right)$.Then go to step 4 .

Step 4 Obtain the decision results of the second stage model (6) using Genetic algorithm

Step 4.1 Construct the population size $N$ and generate the initial population;

Step 4.2 Compute the fitness value $p(x)=\sum_{k=1}^{s} w_{k} p_{k}\left(x_{0}\right)$;

Step 4.3 Set crossover probability $p_{c}$, mutation probability $p_{m}$, the maximum iteration number Iter $_{\max }$, then implement a crossover operation and mutation operation;

Step 4.4 After Iter ${ }_{\max }$ iterations, the best solution is obtained. [End]

\section{THE APPLICATION ANALYSIS OF TS-MOPM}

\subsection{Case Study}

In this section, we will analyze the characteristics and test the effectiveness of TS-MOPM through a real case of multi-objective programming.

Case description: A pharmaceutical factory wants to produce two kinds of granular drugs $\mathrm{M}$ and $\mathrm{N}$ by using one raw material. According to previous statistics, the time required to produce 1 ton of $\mathrm{M}$ and $\mathrm{N}$ is $\gamma_{1}$ hours and $\gamma_{2}$ hours respectively $\left(\gamma_{1} \sim N(2,0.01)\right.$, $\left.\gamma_{2} \sim N(4,0.04)\right)$, the required raw materials are $\eta_{1}$ tons and $\eta_{2}$ tons respectively $\left(\eta_{1} \sim N(3,0.04), \eta_{2} \sim N(5,0.09)\right)$. Additionally, the sales profit (dollars/ton) of the two drugs are slightly different in different regions. According to the data, the approximate sales profit distribution is $\xi_{1} \sim N\left(4000,100^{2}\right)$ and $\xi_{2} \sim N\left(3200,90^{2}\right)$. The market demand for $\mathrm{M}$ is greater than that for $\mathrm{N}$ but is not more than 1.5 times. In the next production cycle, equipment time is not more than 120 hours as possible and overtime is not expected; the total raw materials use should not exceed 150 tons as possible. In the profit aspect, the total profits are expected to be more than 150000 dollars as possible. Please try to make an appropriate periodic production plan for this pharmaceutical factory.

Set $x_{1}, x_{2}$ be the production quantities of drugs $M$ and $\mathrm{N}$ respectively, $X_{1}, X_{2}, X_{3}$ be the total equipment occupancy time, total material consumption and total profit respectively, then we have $X_{1}=\gamma_{1} x_{1}+\gamma_{2} x_{2} \sim N\left(2 x_{1}+4 x_{2}, 0.01 x_{1}^{2}+0.04 x_{2}^{2}\right), X_{2}=\eta_{1} x_{1}+$ $\eta_{2} x_{2} \sim N\left(3 x_{1}+5 x_{2}, 0.04 x_{1}^{2}+0.09 x_{2}^{2}\right), X_{3}=\xi_{1} x_{1}+\xi_{2} x_{2} \sim$ $N\left(4000 x_{1}+3200 x_{2}, 100^{2} x_{1}^{2}+90^{2} x_{2}^{2}\right)$. If $X_{1}, X_{2}, X_{3}$ are viewed as 
the decision objectives of drug manufacture, then this problem can be summarized as the following multi-objective programming:

$$
\begin{aligned}
& \min f\left(x_{1}, x_{2}\right)=\left\{f_{1}\left(X_{1}\right), f_{2}\left(X_{2}\right), f_{3}\left(X_{3}\right)\right\} \\
& \text { s.t. }\left\{\begin{array}{l}
x_{1}-1.5 x_{2} \leq 0 \\
x_{1}-x_{2} \geq 0 \\
x_{1}, x_{2} \geq 0
\end{array}\right.
\end{aligned}
$$

In the following, we will give the solution of model (11) by TSMOPM.

I The first stage model. Because equipment occupancy time $X_{1}$, raw materials $X_{2}$, and total profit $X_{3}$ are random variables obeying normal distribution, they can be quantified through expectation values, i.e., $E\left(f_{1}\left(X_{1}\right)\right)=\left\{2 x_{1}+4 x_{2}, 120\right\}, E\left(f_{2}\left(X_{2}\right)\right)=$ $\left\{3 x_{1}+5 x_{2}, 150\right\}, E\left(f_{3}\left(X_{3}\right)\right)=\left\{4000 x_{1}+3200 x_{2}, 150000\right\}$. Let $d_{1}^{+}, d_{1}^{-}, d_{2}^{+}, d_{2}^{-}, d_{3}^{+}, d_{3}^{-}, w_{1}, w_{2}, w_{3}$ denote the positive, negative deviations and weight coefficients of three targets respectively, we can get the following model:

$$
\begin{aligned}
& \min z=w_{1}\left(d_{1}^{-}+d_{1}^{+}\right)+w_{2} d_{2}^{+}+w_{3} d_{3}^{-} \\
& \text {s.t. }\left\{\begin{array}{l}
2 x_{1}+4 x_{2}+d_{1}^{-}-d_{1}^{+}=120 \\
3 x_{1}+5 x_{2}+d_{2}^{-}-d_{2}^{+}=150 \\
4000 x_{1}+3200 x_{2}+d_{3}^{-}-d_{3}^{+}=150000 \\
x_{1}-1.5 x_{2} \leq 0 \\
x_{1}-x_{2} \geq 0 \\
x_{1}, x_{2} \geq 0, d_{i}^{+}, d_{i}^{-} \geq 0, i=1,2,3
\end{array}\right.
\end{aligned}
$$

Use lingo to solve (12), the solution results are stated as Table 1 in the following:

Table 1 presents all the satisfactory solutions with different objective weights. We can see that, the deviations of total profits are 0 as the

Table 1 Results obtained from the first stage.

\begin{tabular}{cccccccc}
\hline \multicolumn{2}{c}{ Parameter } & $\boldsymbol{x}_{\mathbf{1}}$ & $\boldsymbol{x}_{\mathbf{2}}$ & $\boldsymbol{d}_{\mathbf{1}}^{-}$ & $\boldsymbol{d}_{\mathbf{1}}^{+}$ & $\boldsymbol{d}_{\mathbf{2}}^{+}$ & $\boldsymbol{d}_{\mathbf{3}}^{-}$ \\
\hline \multirow{2}{*}{ Case 1 } & $w_{1}=0.8$ & & & & & & \\
& $w_{2}=0.1$ & 22.5 & 18.75 & 0 & 0 & 11.25 & 0 \\
& $w_{3}=0.1$ & & & & & & \\
Case 2 & $w_{1}=0.1$ & & & & & & \\
& $w_{2}=0.8$ & 24.4565 & 16.3044 & 5.8696 & 0 & 4.8913 & 0 \\
& $w_{3}=0.1$ & & & & & & \\
Case 3 & $w_{1}=0.1$ & & & & & & \\
& $w_{2}=0.1$ & 24.4565 & 16.3044 & 5.8696 & 0 & 4.8913 & 0 \\
& $w_{3}=0.8$ & & & & & & \\
Case 4 & $w_{1}=0.4$ & & & & & & \\
& $w_{2}=0.4$ & 24.4565 & 16.3044 & 5.8696 & 0 & 4.8913 & 0 \\
& $w_{3}=0.2$ & & & & & & \\
Case 5 & $w_{1}=0.4$ & & & & & & \\
& $w_{2}=0.2$ & 22.5 & 18.75 & 0 & 0 & 11.25 & 0 \\
& $w_{3}=0.4$ & & & & & & \\
Case 6 & $w_{1}=0.2$ & & & & & & \\
& $w_{2}=0.4$ & 24.4565 & 16.3044 & 5.8696 & 0 & 4.8913 & 0 \\
& $w_{3}=0.4$ & & & & & & \\
Case 7 & $w_{1}=1 / 3$ & & & & & & \\
& $w_{2}=1 / 3$ & 24.4565 & 16.3044 & 5.8696 & 0 & 4.8913 & 0 \\
& $w_{3}=1 / 3$ & & & & & & \\
\hline
\end{tabular}

weights change. It is because that "the magnitude of profit is larger than the other two targets values." This fact leads to no significant changes of the solution results. Moreover, the results have the following two shortcomings: (1) Taking the minimum absolute deviation as the objective, the reliability of the decision-making results cannot be demonstrated, which is an important index evaluating the decision-making quality; (2) The required decision-making objectives have different dimensions. Therefore, it is difficult to explain the practical meaning of the decision-making results explicitly.

II The second stage model. According to Theorem 1 and Remark 2, the satisfactory state value of the equipment occupy time can be expressed as $V_{1}=(110,120,130)$; the minimum total raw materials consumption is 0 , so its satisfactory state value can be expressed as $V_{2}=(0,150,150) \cup[150,150,160)$; the total profit can be $V_{3}=(145000,150000,150000) \cup[150000,150000, \infty)$. Based on the probability calculation of fuzzy events, then the following multi-objective programming model with the maximal synthetic realization probability of each objective is established:

$$
\begin{aligned}
& \max p\left(x_{1}, x_{2}\right)=w_{1} \cdot p_{1}\left(x_{1}, x_{2}\right)+w_{2} \cdot p_{2}\left(x_{1}, x_{2}\right)+w_{3} \cdot p_{3}\left(x_{1}, x_{2}\right) \\
& \text { s.t. }\left\{\begin{array}{l}
p_{k}\left(x_{1}, x_{2}\right)=P\left(f_{k}(x) \in V_{k}\right) \\
x_{1}-1.5 x_{2} \leq 0 \\
x_{1}-x_{2} \geq 0 \\
\sum_{k=1}^{3} w_{k} p_{k}\left(x_{1}, x_{2}\right) \geq \beta \\
x_{1}, x_{2} \geq 0 ; k=1,2,3
\end{array}\right.
\end{aligned}
$$

According to Theorem 2, it is difficult to determine the numerical computation of $p_{k}\left(x_{1}, x_{2}\right)$. Therefore, there does not exist formalized solution methods for model (13). Under this case, we can compute the probability of each objective using MATLAB programming. If $x_{1}=22.5, x_{2}=18.75$, then the probability can be $p_{1}=$ $0.6544, p_{2}=0.2109, p_{3}=0.8201$; if $x_{1}=24.4565, x_{2}=16.3044$, then $p_{1}=0.4188, p_{2}=0.5057, p_{3}=0.7816$. When different weights are given to each objective, the maximum synthetic realization degree $\beta$ under different decision-making preferences can then be determined.

We use GA to obtain various results under different decisionmaking preference listed in Table 2. Particularly, GA works with binary-encoded strings with length 20 . Set the size of population as 80 , crossover probability as 1 , mutation probability as 0.001 , and maximal iterations as 100 .

From Table 2, we know that, (1) Compared with Table 1, the decision-making results of Table 2 are more sensitive to the change of weight, which shows that the attention degree of each objective will directly affect the decision-making results; (2) Under different decision-making states, the synthetic realization degree of the objectives in the second stage is higher than that in the first stage. Occasionally, the synthetic realization degree is the same as case 2. All these indicate that TS-MOPM has higher reliability than expectation value model in decision-making results. So TS-MOPM has important reference value and instructive guide; (3) TS-MOPM has good interpretability. Both the synthetic reliability of decisionmaking results with different preferences and the realization degree 
Table 2 Results obtained from the second stage.

\begin{tabular}{|c|c|c|c|c|c|c|c|c|}
\hline \multicolumn{2}{|c|}{ Parameters } & $\beta$ & $x_{1}$ & $x_{2}$ & $p_{1}\left(x_{1}, x_{2}\right)$ & $p_{2}\left(x_{1}, x_{2}\right)$ & $p_{3}\left(x_{1}, x_{2}\right)$ & $p\left(x_{1}, x_{2}\right)$ \\
\hline Case 1 & $\begin{array}{l}w_{1}=0.8 \\
w_{2}=0.1 \\
w_{3}=0.1 \\
w_{1}=0.1\end{array}$ & 0.6266 & 24.7067 & 17.6442 & 0.6593 & 0.1737 & 0.9917 & 0.6440 \\
\hline Case 2 & $\begin{array}{l}w_{2}=0.8 \\
w_{3}=0.1\end{array}$ & 0.5246 & 24.4565 & 16.3044 & 0.4188 & 0.5057 & 0.7816 & 0.5246 \\
\hline Case 3 & $\begin{array}{l}w_{2}=0.1 \\
w_{3}=0.8 \\
w_{1}=0.4\end{array}$ & 0.7171 & 24.5601 & 17.8299 & 0.6559 & 0.1587 & 0.9918 & 0.8749 \\
\hline Case 4 & $\begin{array}{l}w_{2}=0.4 \\
w_{3}=0.2\end{array}$ & 0.5261 & 24.6090 & 17.0127 & 0.6037 & 0.3062 & 0.9516 & 0.5543 \\
\hline Case 5 & $\begin{array}{l}w_{1}=0.4 \\
w_{2}=0.2 \\
w_{3}=0.4 \\
w_{1}=0.2\end{array}$ & 0.6320 & 24.6090 & 17.4770 & 0.6551 & 0.2120 & 0.9827 & 0.6975 \\
\hline Case 6 & $\begin{array}{l}w_{2}=0.4 \\
w_{3}=0.4\end{array}$ & 0.5987 & 24.8534 & 16.8915 & 0.6042 & 0.3006 & 0.9669 & 0.6278 \\
\hline Case 7 & $\begin{array}{l}w_{1}=1 / 3 \\
w_{2}=1 / 3 \\
w_{3}=1 / 3\end{array}$ & 0.5687 & 25.0 & 16.8456 & 0.6089 & 0.2914 & 0.9755 & 0.6253 \\
\hline
\end{tabular}

of each objective can be directly obtained, which is beneficial for the explanation of the implications of the results under different states.

\subsection{Other Applications}

Two stage multi-objective programming techniques under random environment have been widely applied to handle many real decision problems.

1. Transportation network design. In recent years, as the travel demands rise continuously, severe traffic congestion and transportation delays are often incurred. Optimize transportation network is becoming urgent problems in urban transportation planning. In regard to designing traffic network, transportation cost is of vital importance in the overall transportation process. Additionally, with the development of resource-efficient and sustainable cities, high efficiency and environmental protection are also the main concerns strived for in decision-making process. Therefore, transportation network design can be posed as a multi-objective programming problem. Transportation cost, transportation time and emissions are three critical aspects that management must minimize to optimize a network. Nevertheless, the randomness of transportation requirements cause the uncertainty of three indexes in an actual integrated freight system. In most cases, three objectives are not of equal importance. In a word, the mentioned problem can be solved through establishing a two stage multi-objective programming model.

2. Supplier selection and management. Supply chain management requires harmonizing the business processes among the members, i.e., suppliers, producers, and distributors. In most industries, the cost of raw material constitutes a large portion of products price. Therefore, to reduce the production cost of a product, it is increasingly important to select appropriate suppliers which can directly influence the efficiency of the business [7]. Additionally, the ever changing complexity of competitive environment makes cost minimization not the only objective, other factors such as the quality of raw materials and on-time delivery are of great significance. Nevertheless, all the indexes "cost minimization, good quality and on-time delivery" have uncertainty. Coupled with the variability of business environment, the supplier selection problem can be investigated through two stage multi-objective programming model.

\section{CONCLUSIONS}

For random numerical multi-objective decision-making problems, this paper extends the previous researches and establishes TS-MOPM by taking fuzzy decision-making objective as fuzzy events. TS-MOPM has the following advantages:

First, TS-MOPM uses probability to describe all the decisionmaking objectives. It can eliminate the dimensional influence on the explanation of decision-making results, and make up for the deficiencies of priority factor model to some extent. Moreover, the probability calculation of fuzzy events has generality. Therefore, TS-MOPM has general applications.

Second, TS-MOPM tells the decision-makers the realization degree of decision-making results intuitively. Even if the randomness is even greater, the decision-making results can also be guaranteed. It makes up for the deficiencies of expectation value model.

Third, TS-MOPM has better operability and solvability. It can avoid high computation complexity due to too many decision-making objectives and no solution due to inappropriate confidence level for chance- constrained programming model.

The probability calculation involved in TS-MOPM is a key issue in its application. Although Section 4 gives several methods of 
some special fuzzy events, these work cannot fairly meet decisionmaking needs in complex random environment. Thus, further work is needed to construct probability calculation platform based on stochastic simulation and decision-making system based on probability.

\section{CONFLICT OF INTEREST}

We declare that we do not have any commercial or associative interest that represents a conflict of interest in connection with the work.

\section{AUTHORS' CONTRIBUTIONS}

Chenxia Jin: Conceptualization, Writing - review \& editing. Fachao Li: Visualization. Kaixin Feng: Writing - original draft. Yunfeng Gup: Software.

\section{ACKNOWLEDGMENTS}

This work is supported by the National Natural Science Foundation of China $(71771078,71371064)$ and Graduate research and innovation projects of Hebei Province (CXZZBS2020076).

\section{REFERENCES}

[1] Q.Q. Rong, Y.P. Cai, M.R. Su, W.C. Yue, Z.F. Yang, Z. Dang, A simulation-based bi-level multi- objective programming model for watershed water quality management under interval and stochastic uncertainties, J. Environ. Manag. 245 (2019), 418-431.

[2] Q.Q. Li, Y.P. Li, G.H. Huang, C.X. Wang, Risk aversion based interval stochastic programming approach for agricultural water management under uncertainty, Stoch. Environ. Res. Risk A. 32 (2018), 715-732.

[3] S. Tofighi, S.A. Torabi, S.A. Mansouri, Humanitarian logistics network design under mixed uncertainty, Eur. J. Oper. Res. 250 (2016), 239-250.

[4] J.H. Zhang, H.Y. Liu, G.D. Yu, J.H. Ruan, F.T.S. Chan, A threestage and multi-objective stochastic programming model to improve the sustainable rescue ability by considering secondary disasters in emergency logistics, Comput. Ind. Eng. 135 (2019), 1145-1154.

[5] Y.C. Chen, D.J. Guo, Z.L. Chen, Y.Q. Fan, X. Li, Using a multiobjective programming model to validate feasibility of an underground freight transportation system for the Yangshan port in Shanghai, Tunn. Undergr. Space Technol. 81 (2018), 463-471.

[6] C.F. Ren, Z.H. Li, H.B. Zhang, Integrated multi-objective stochastic fuzzy programming and AHP method for agricultural water and land optimization allocation under multiple uncertainties, J. Clean. Prod. 210 (2019), 12-24.

[7] E.B. Tirkolaee, A. Mardani, Z. Dashtian, M. Soltani, G.W. Weber, A novel hybrid method using fuzzy decision making and multiobjective programming for sustainable-reliable supplier selection in two- echelon supply chain design, J. Clean. Prod. 250 (2020), 119517.
[8] G.H. Tzeng, J.J. Huang, Fuzzy Multiple Objective Decision Making, CRC Press, Boca Raton, Florida, USA, 2014, pp. 6-7.

[9] J. Lu, J.L. Han, Y.G. Hu, G.Q. Zhang, Multilevel decision-making: a survey, Inf. Sci. 346-347 (2016), 463-487.

[10] G.Q. Zhang, J.L. Han, J. Lu, Fuzzy bi-level decision-making techniques: a survey, Int. J. Comput. Intell. Syst. 9 (2016), 25-34.

[11] N. Boland, H. Charkhgard, M. Savelsbergh, Preprocessing and cut generation techniques for multi- objective binary programming, Eur. J. Oper. Res. 274 (2019), 858-875.

[12] A. Charnes, W.W. Cooper, Management model and the industrial applications of linear programming, Manag. Sci. 4 (1957), 38-91.

[13] M. Ehrgott, Multicriteria Optimization, second ed., Springer, New York, NY, USA, 2005.

[14] J.J. Liu, W.P. Wang, X.B. Li, T. Wang, S.Y. Bai, Y.F. Wang, Solving a multi-objective mission planning problem for UAV swarms with an improved NSGA-III algorithm, Int. J. Comput. Intell. Syst. 11 (2018), 1067-1081.

[15] J.L. Han, G.Q. Zhang, Y.G. Hu, J. Lu, A solution to bi/tri-level programming problems using particle swarm optimization, Inf. Sci. 370-371 (2016), 519-537.

[16] J.L. Han, J. Lu, Y. G. Hu, G.Q. Zhang, Tri-level decision-making with multiple followers: model, algorithm and case study, Inf. Sci. 311 (2015), 182-204.

[17] A. Guerrero-Enamorado, C. Morell, S. Ventura, A gene expression programming algorithm for discovering classification rules in the multi-objective space, Int. J. Comput. Intell. Syst. 11 (2018), 540-559.

[18] H. Kao, C.Y. Chan, D.J. Wu, A multi-objective programming method for solving network DEA, Appl. Soft Comput. 24 (2014), 406-413.

[19] Y. Dujardin, D. Vanderpooten, F. Boillot, A multi-objective interactive system for adaptive traffic control, Eur. J. Oper. Res. 244 (2015), 601-610.

[20] Y. Zhou, L.J. Kong, Z.Y. Wu, S.P. Liu, Y.Q. Cai, Y. Liu, Ensemble of multi-objective metaheuristic algorithms for multi-objective unconstrained binary quadratic programming problem, Appl. Soft Comput. J. 81 (2019), 105485.

[21] A. Pal, H. Charkhgard, FPBH: a feasibility pump based heuristic for multi-objective mixed integer linear programming, Comput. Oper. Res. 112 (2019), 104760.

[22] B. Lokman, M. Köksalan, P.J. Korhonen, J. Wallenius, An interactive approximation algorithm for multi-objective integer programs, Comput. Oper. Res. 96 (2018), 80-90.

[23] B.D. Liu, R.Q. Zhao, Stochastic programming and fuzzy programming, Tsinghua University Press, Beijing, China, 1998.

[24] A. Charnes, W.W. Cooper, Chance-constrained programming, Manag. Sci. 6 (1959), 73-79.

[25] B.D. Liu, Dependent-chance programming: a class of stochastic programming, Comput. Math. Appl. 34 (1997), 89-104.

[26] F.B. Abdelaziz, B. Aouni, R.E. Fayedh, Multi-objective stochastic programming for portfolio selection, Eur. J. Oper. Res. 177 (2007), 1811-1823.

[27] F.C. Li, C.X. Jin, L. Wang, Quasi-linear stochastic programming model based on expectation and variance and its application in transportation problem, Appl. Math. Model. 38 (2014), 1919-1928.

[28] Z. Sazva, S.M.J. Mirzapour Al-e-hashem, A. Baboli, M.R. Akbari Jokar, A bi-objective stochastic programming model for a centralized green supply chain with deteriorating products, Int. J. Prod. Econ. 150 (2014), 140-154. 
[29] H. Masri, Y. Abdulla, A multiple objective stochastic programming model for working capital management, Technol. Forecast. Soc. Change. 131 (2018), 141-146.

[30] L.Z. Tong, R.X. Niu, Y. He, Model in design of Logistics network based on stochastic multiple objectives, Logistics Technol. 33 (2014), 254-256.

[31] A. Fard, M. Hajiaghaei-Keshteli, A stochastic multi-objective model for a closed-loop supply chain with environmental considerations, Appl. Soft Comput. 69 (2018), 232-249.

[32] J. Nematian, S.R. Movahhed, An extended multi-objective mixed integer programming for water resources management through probability theory, Ecol. Inform. 54 (2019), 100992.
[33] L.A. Zadeh, Fuzzy sets, Inf. Control. 8 (1965), 338-353.

[34] L.A. Zadeh, Probability measures of fuzzy events, J. Math. Anal. Appl. 23 (1968), 421-427.

[35] R.R. Yager, A note on probabilities of fuzzy events, Inf. Sci. 18 (1979), 113-122.

[36] R.R. Yager, A representation of the probability of a fuzzy subset, Fuzzy Sets Syst. 13 (1984), 273-283.

[37] D.G. Chen, W.X. Yang, F.C. Li, Measures of general fuzzy rough sets on a probabilistic space, Inf. Sci. 178 (2008), 3177-3187.

[38] F.C. Li, J.D. Jie, Research on effect probability measurement of fuzzy events, ICIC Express Lett. Part B Appl. 6 (2015), 2167-2176. 